\title{
Electroacupuncture Improves Cognitive Deficits through Increasing Regional Cerebral Blood Flow and Alleviating Inflammation in CCI Rats
}

\author{
Dexiong Han, ${ }^{1}$ Zhe Liu, ${ }^{2}$ Gaimei Wang, ${ }^{1}$ Ying Zhang, ${ }^{1}$ and Zemin $\mathrm{Wu}^{2}$ \\ ${ }^{1}$ The 3rd Affiliated Hospital of Zhejiang Chinese Medical University, Hangzhou 310005, China \\ ${ }^{2}$ Zhejiang Chinese Medical University, Hangzhou 310053, China \\ Correspondence should be addressed to Zhe Liu; ssrsliu@163.com
}

Received 28 October 2016; Revised 1 January 2017; Accepted 15 March 2017; Published 10 April 2017

Academic Editor: Cheorl-Ho Kim

Copyright (C) 2017 Dexiong Han et al. This is an open access article distributed under the Creative Commons Attribution License, which permits unrestricted use, distribution, and reproduction in any medium, provided the original work is properly cited.

\begin{abstract}
Objective. To investigate the effect of EA on regional cerebral blood flow, cognitive deficits, inflammation, and its probable mechanisms in chronic cerebral ischemia (CCI) rats. Methods. Rats were assigned randomly into sham operation group (sham group) and operation group. For operation group, CCI model was performed using the permanent bilateral common carotid artery occlusion (BCCAO) method, and then rats were further randomly divided into model group and electroacupuncture (EA) group. 2/15 Hz low-frequency pulse electric intervention was applied at "Baihui" and "Dazhui" acupoints in EA group. Four weeks later, Morris water maze test was adopted to assess the cognitive function, using laser Doppler flowmetry to test changes of regional cerebral blood flow ( $\mathrm{rCBF}$ ); double antibody sandwich enzyme-linked immunosorbent assay (DAS-ELISA) to measure proinflammatory cytokines (IL-6, TNF- $\alpha$, and IL-1 $\beta$ ); western blot to test the protein expression quantities of proinflammatory cytokines, JAK2, and STAT3; and RT-PCR to test JAK2 mRNA and STAT3 mRNA in the hippocampus in each group. Results. Compared with the model group, learning and memory abilities and $\mathrm{rCBF}$ and IL- 6 expression of the EA group enhanced markedly; IL-1 $\beta$ and JAK2 significantly decreased; TNF- $\alpha$ and STAT3 also declined, but the difference was not apparent. Conclusion. Our research suggests that EA can improve cognitive deficits which may be induced by increasing rCBF and anti-inflammatory effect.
\end{abstract}

\section{Introduction}

Long-lasting decrease in cerebral blood flow caused by chronic cerebral ischemia (CCI) is a key etiological factor of cognitive dysfunction among the aged [1-3]. The underlying pathological mechanisms behind CCI remain unclear. Previous studies have identified several neuropathological changes including immune inflammatory injury [4], oxidative stress injury [5], synaptic structural and functional disorders [6], neurotransmission disorders of the central cholinergic and monoaminergic system $[7,8]$, and white matter injury [9]. Permanent bilateral common carotid artery occlusion (BCCAO) model has been widely used in CCI because it can provide a moderate but persistent reduction in regional cerebral blood flow ( $\mathrm{rCBF}$ ) which compromises memory processes and contributes to the development and progression of various cognitive disorders [7].
Inflammation in the CNS occurs partly through the release of numerous factors like cytokines, chemokines, or growth factors, leading to a complex crosstalk between different brain cell types [10]. Inflammatory reaction, which is induced following the BCCAO in the white matter and hippocampus $[3,11]$, plays a crucial role in chronic ischemic cerebrovascular diseases [12]. The activation of JAK-STAT signaling pathway is an important component of the factors released during inflammation. Interleukin 1 (IL-1), IL-6, and tumor necrosis factor-alpha (TNF- $\alpha$ ) are most widely studied [13] among the numerous inflammatory factors. Some of these inflammatory factors play synergistic roles in inflammatory injury, thereby aggravating cerebral ischemic injury and gradually deteriorating the cognitive function. Studies revealed that, during CCI injury process, electroacupuncture (EA) could upregulate vascular endothelial growth factor expression, promote the generation of new vessels, and 
improve neuronal function $[14,15]$. However, these results are insufficient to elucidate the multitarget and multichannel mechanism of acupuncture. Thus, this study aims to investigate the potential mechanisms of EA on CCI from the aspect of increasing $\mathrm{rCBF}$ and preventing inflammation.

\section{Materials and Methods}

2.1. Animals. Animal care, operation, treatment procedures, and animal welfare were executed in strict accordance with the National Institutions of Health Guide for the Care and Use of Laboratory Animals by the Experimental Animal Center of Zhejiang Chinese Medical University. Healthy male Sprague-Dawley (SD) rats (weight $200 \pm 20 \mathrm{~g}$ and ages 3-6 months) were brought from and fed in the Experimental Animal Center of Zhejiang Chinese Medical University. They were placed in standard cages under a specific circumstance where light-dark cycle is $12 \mathrm{~h} / 12 \mathrm{~h}$, relative humidity is $45 \%-50 \%$, and temperature is $22 \pm 2^{\circ} \mathrm{C}$. The enrolled rats first underwent the Morris water maze test to exclude those with low intelligence and poor swimmers to ensure that the cognitive function did not show any significant differences among all rats before the study.

\subsection{Animal Modeling and Evaluation}

Operation Group. CCI model was performed by BCCAO method [16]. In brief, under $10 \%$ chloral hydrate $(40 \mathrm{mg} / \mathrm{kg}$, i.p.) anesthesia, bilateral common carotid arteries of the rat were carefully separated from the cervical sympathetic and vagal nerves through a ventral cervical incision. Then, the arteries were doubly ligated with 5/0 silk sutures simultaneously. The skin incision was closed and the rats were kept in an air-conditioned room as described previously. Before the operation, the rats were fastened for $12 \mathrm{~h}$ and deprived of water for $4 \mathrm{~h}$.

Sham Group. The rats were treated in the same manner as the operation group except for the bilateral common carotid artery occlusion.

Two hours after the rat recovered from anesthesia, two rats were randomly selected from respective operation group and sham operation group to evaluate $\mathrm{rCBF}$ and then killed and their brains were sliced for pathological evaluation to ensure that the rat model of CCI was successfully copied.

2.3. Grouping. After modeling, rats of the operation group were further assigned randomly into the model group and EA group, each consisting of 10 rats. The sham group also comprised 10 rats.

2.4. EA Treatment. Rats of the EA group receive EA therapy. In the whole procedure, slip eyeshades over rats' eyes to keep them relatively comfortable and quiet. "Baihui" (DU20) and "Dazhui" (DU14) acupoints were selected according to the Acupoint Standard for Experimental Animals issued by the Society of Experimental Acupuncture, China Association for Acupuncture and Moxibustion in 1992. Acupuncture was performed using the following instruments and parameters: needle: $0.25 \mathrm{~mm}$ in diameter $\times 25 \mathrm{~mm}$ in length Huatuo stainless steel filiform needle (Suzhou Medical Supplies Co., Ltd., China) and HAN's Acupoint Nerve Stimulator (Nanjing Jisheng Medical Treatment Technology Co., Ltd., China): $2 / 15 \mathrm{~Hz}$ dilatational wave of frequency with automatically shifting between $2 \mathrm{~Hz}$ and $15 \mathrm{~Hz}$ stimulation for $3 \mathrm{~s}$ each, with current intensity $2 \pm 1 \mathrm{~mA}$ (causing slight vibration of muscles around acupoints and keeping quiet), lasting for $30 \mathrm{~min}$, once in the morning, five times every week, with break at weekend, and the treatment continued for 4 weeks.

\subsection{Evaluation Indicators and Testing Methods}

2.5.1. Evaluation of Cognitive Function. Cognitive function of various groups was evaluated by Morris water maze system (Beijing Taiji Electronic Co., Ltd., Beijing, China).

(1) Navigation Test. The test started from the last week of the treatment. After EA therapy was completed in the EA group, rats of the three groups underwent a navigation test, twice daily, with an interval of $15 \mathrm{~min}$, for successive 5 days, and the average values were adopted. Experimental data and images were analyzed using an image automatic monitoring and processing system.

(2) Probe Trial. The trial was conducted on the fifth day after conducting the navigation test. The platform in the pool was removed, and the rats were allowed to swim freely, where the entrance was located at the contralateral quadrant of the platform. The memory function was evaluated using the time for the rats to cross the quadrant of the former platform within $120 \mathrm{~s}$. This trial was performed twice, with an interval of $15 \mathrm{~min}$.

2.5.2. $r$ CBF Test. rCBF was tested before and after BCCAO (within $30 \mathrm{~min}$ ) and before killing by laser Doppler flowmetry (PeriFLUX5000, Sweden) including the following steps. Right temporal side of the brain of rats [14] (midpoint of the outer canthus and the earhole) was selected as the testing point. Hair was shaved, skin was disinfected with alcohol and polyvinyl iodine, and then a $5 \mathrm{~mm}$ longitudinal incision was made. The muscles were bluntly disserted to expose the bone surface, which was cleaned with sterile cotton balls dipped in $3 \% \mathrm{H}_{2} \mathrm{O}_{2}$. Then the probe base was fixed onto the bone surface using specific glue. After this, the probe was inserted into the base, and these data were recorded and stored after they were stabilized on the computer monitor (recording time $\geq$ $3 \mathrm{~min}$ ). Rats were anesthetized with ether in specimen jar for about 60-90 seconds to be just unconscious before test each time.

2.5.3. DAS-ELISA. Rats were rapidly sacrificed after anesthesia with chloral hydrate after the last rCBF test. Concentrations of IL- 6 , TNF- $\alpha$, and IL- $1 \beta$ in the hippocampus of rats were determined by DAS-ELISA according to the manufacturer's instructions (Shanghai Westang BioTech Inc., Ltd., Shanghai, China). 
2.5.4. Western Blot. Western blot was performed to measure protein expression of IL-6, TNF- $\alpha$, IL-1 $\beta$, JAK2, and STAT3. Hippocampus tissues were homogenized in strong RIPA buffer. The homogenate was allowed to rest on ice for $30 \mathrm{~min}$ and then centrifuged at $15,000 \mathrm{rpm}$ for $15 \mathrm{~min}$ at $4^{\circ} \mathrm{C}$, and the supernatant was collected. The protein concentration of tissue lysates was determined with a BCA protein assay kit. The lysates were separated on 10\% SDS-PAGE gel and transferred to polyvinylidene difluoride (PVDF) membranes (Bio-Rad, Hercules, CA, USA). The membranes were blocked with 5\% nonfat powdered milk in TBST (Tris-buffered saline containing $0.1 \%$ Tween 20 ) for $1 \mathrm{~h}$ at room temperature (RT) and then incubated overnight at $4^{\circ} \mathrm{C}$ with the following primary antibodies: IL-6 (Abcam); IL-1 $\beta$ (Millipore); TNF- $\alpha$ (Santa Cruz); JAK2 (Sigma-Aldrich), STAT3 (Sigma-Aldrich), $\beta$ actin (Abcam). After washing in TBST, the membrane was incubated for $1 \mathrm{~h}$ at RT with HRP-conjugated goat anti-rabbit antibody (West Grove), and protein bands were visualized using the Immun-Star ${ }^{\mathrm{TM}}$ HRP Chemiluminescence Kit (Bio$\mathrm{Rad}$ ). Images of bands were recorded by the ImageQuant LAS 4000 system (GE Healthcare) and the band intensities were quantified using ImageQuant TL software (version 7.0, GE Healthcare). $\beta$-Actin was used as the internal loading control.

2.5.5. RT-PCR. Total RNA was isolated from hippocampus by TriBlue RNA kit (Ambion, USA) according to the manufacture's protocol. $1 \mu \mathrm{g}$ of total RNA was used as a template for reverse transcription using. PCR amplification of the JAK2, STAT3, and $\beta$-actin was as follows: JAK2, sense: $5^{\prime}$-GTTCTTACCGAAGTGCGTGCGA-3' ${ }^{\prime}$, antisense: $5^{\prime}$-GGTAATGGTGTGCATCCGCAGTT-3' ${ }^{\prime}$; STAT3, sense: $5^{\prime}$-TGGAAGAGGCGGCAGCAGATAGC-3' , antisense: $5^{\prime}$-CACGGCCCCCATTCCCACAT- $3^{\prime} ; \beta$-actin, sense: $5^{\prime}$-TCAGGTCATCACTATCGGCAAT- ${ }^{\prime}$, antisense: $5^{\prime}$-AAAGAAAGGGTGTAAAACGCA- $3^{\prime}$. The thermal cycling parameters were as follows: JAK2, $95^{\circ} \mathrm{C}$ for $5 \mathrm{~min}$, followed by 30 cycles of amplification at $95^{\circ} \mathrm{C}$ for $20 \mathrm{~s}$, and $72^{\circ} \mathrm{C}$ for $45 \mathrm{~s}$; STAT3, $95^{\circ} \mathrm{C}$ for $5 \mathrm{~min}$, followed by 30 cycles of amplification at $95^{\circ} \mathrm{C}$ for $20 \mathrm{~s}$, and $60^{\circ} \mathrm{C}$ for $30 \mathrm{~s} ; \beta$-actin, $95^{\circ} \mathrm{C}$ for $5 \mathrm{~min}$, followed by 30 cycles of amplification at $94^{\circ} \mathrm{C}$ for $30 \mathrm{~s}$, and $72^{\circ} \mathrm{C}$ for $30 \mathrm{~s}$. The amplification products were incubated with $\mathrm{EB}$, separated from agarose gel electrophoresis, and detected by GIS Gel image processing system. The average optical density of the bands was analyzed with $\beta$-actin as internal control.

2.6. Statistical Analysis. Statistical analyses were performed by SPSS 16.0 (SPSS, IL, USA). Descriptive data were expressed as mean \pm standard deviation $(\bar{x} \pm s)$. For the navigation test, multiple-factor variance analysis for repeated measurement data was adopted. Intragroup comparisons were performed before and after intervention using pairwise $t$-test and intergroup comparisons were conducted using one-way analysis of variance (ANOVA). In addition, the $t$-test was applied after the data were confirmed with equal variance while the ranksum test was adopted after the data were confirmed with unequal variance. A $P$ value of less than 0.05 was considered statistically.

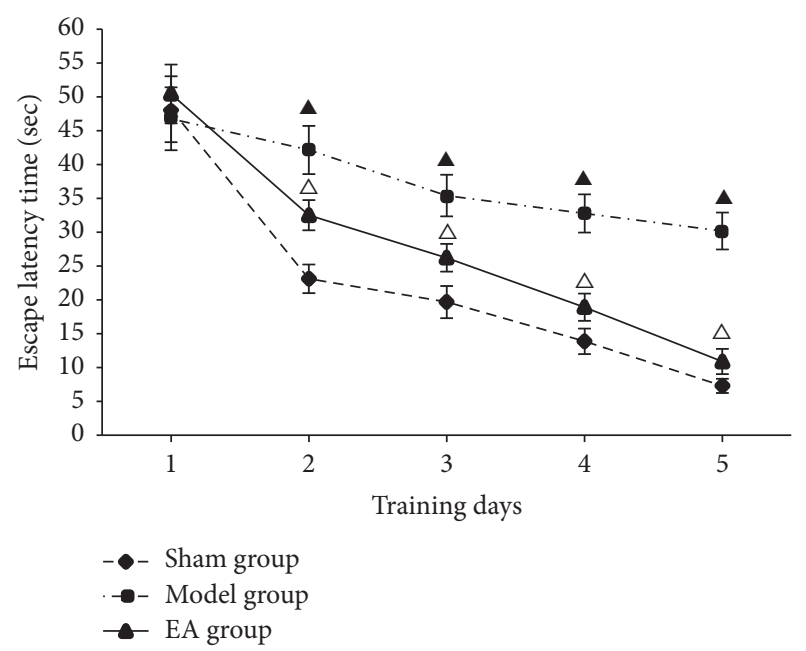

Figure 1: Performance of rats during navigation test. ${ }^{\boldsymbol{\Lambda}} P<0.01$, versus sham group; ${ }^{\triangle} P<0.05$, versus model group.

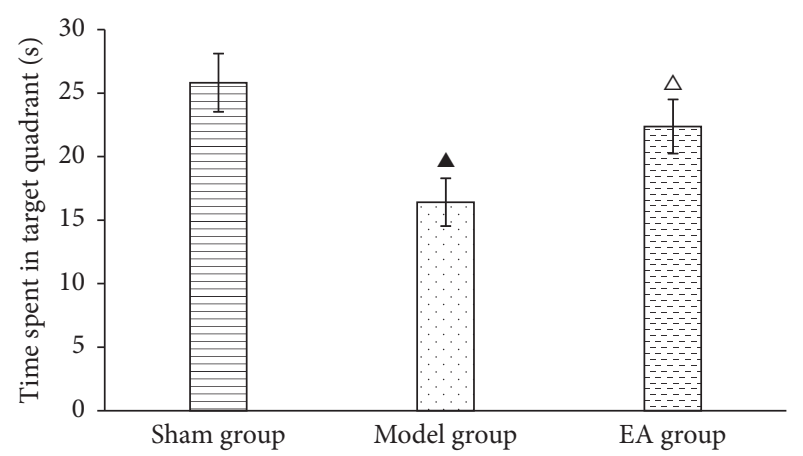

Figure 2: Time spent in the target quadrant of rats. ${ }^{\wedge} P<0.05$, versus sham group; ${ }^{\triangle} P<0.05$, versus model group.

\section{Results}

\subsection{Evaluation of Cognitive Function}

3.1.1. Navigation Test. Navigation test results showed that although escape latency did not have a significant difference among three groups on the first day $(P>0.05)$, after 5-day training, compared with model group, the escape latency of the sham group quickly decreased to a plateau $(P<0.01)$, while that of EA group also significantly declined to a plateau $(P<0.05)$, indicating that EA can improve learning and memory ability in CCI rats (Table 1, Figure 1).

3.1.2. Probe Trial. Time spent in the plateau quadrant of the EA group was longer than that of the model group, with a significant difference between two groups $(P<0.05)$, and close to that of the sham group, suggesting that $\mathrm{EA}$ can improve the memory in CCI rats (Table 2, Figure 2).

\subsection{Changes of $r C B F$}

3.2.1. $r C B F$ beforelafter BCCAO. The $\mathrm{rCBF}$ of pre-BCCAO did not show any significant difference between the sham and 
TABLE 1: Performance of rats during navigation test $(\bar{x} \pm s, s)$.

\begin{tabular}{|c|c|c|c|c|c|}
\hline \multirow{2}{*}{ Groups } & \multicolumn{5}{|c|}{ Training days } \\
\hline & 1 & 2 & 3 & 4 & 5 \\
\hline Sham group & $48.17 \pm 4.87$ & $23.11 \pm 2.12$ & $19.67 \pm 2.38$ & $13.87 \pm 1.89$ & $7.29 \pm 1.05$ \\
\hline Model group & $46.76 \pm 4.65$ & $42.15 \pm 3.57^{\mathrm{a}}$ & $35.42 \pm 3.08^{\mathrm{a}}$ & $32.77 \pm 2.82^{\mathrm{a}}$ & $30.18 \pm 2.73^{\mathrm{a}}$ \\
\hline EA group & $50.43 \pm 4.35$ & $32.51 \pm 2.23^{b}$ & $26.23 \pm 2.05^{\mathrm{b}}$ & $18.92 \pm 2.01^{\mathrm{b}}$ & $10.89 \pm 1.87^{b}$ \\
\hline
\end{tabular}

Compared with the sham group, ${ }^{\mathrm{a}} P<0.01$; compared with the model group, ${ }^{\mathrm{b}} P<0.05$.

TABLE 2: Time spent in the target quadrant $(\bar{x} \pm s)$.

\begin{tabular}{lc}
\hline Groups & Time spent in plateau quadrant $(\mathrm{s})$ \\
\hline Sham group & $25.82 \pm 2.29$ \\
Model group & $16.41 \pm 1.88^{\mathrm{a}}$ \\
EA group & $22.37 \pm 2.13^{\mathrm{b}}$ \\
\hline
\end{tabular}

Compared with sham group, ${ }^{\mathrm{a} P}<0.05$; compared with model group, ${ }^{\mathrm{b}} \mathrm{P}<$ 0.05 .

TABLE 3: Changes of rCBF before/after BCCAO $(\bar{x} \pm s, \mathrm{PU})$.

\begin{tabular}{lcc}
\hline Groups & Before BCCAO & After BCCAO \\
\hline Sham group & $160.85 \pm 14.08$ & $155.89 \pm 13.82$ \\
Operation group & $157.57 \pm 13.93$ & $63.15 \pm 8.06^{\mathrm{a}}$ \\
\hline
\end{tabular}

Compared with the sham group, ${ }^{\mathrm{a}} P<0.01$.

TABLE 4: Changes of rCBF in various groups $(\bar{x} \pm s, \mathrm{PU})$.

\begin{tabular}{lc}
\hline Groups & rCBF \\
\hline Sham group & $156.38 \pm 14.29$ \\
Model group & $89.05 \pm 8.18^{\mathrm{a}}$ \\
EA group & $125.89 \pm 10.38^{\mathrm{bc}}$ \\
\hline
\end{tabular}

Compared with the sham group, ${ }^{\mathrm{a}} P<0.01$; compared with the model group,

${ }^{\mathrm{b}} P<0.05$; compared with the sham group, ${ }^{\mathrm{c}} P<0.05$.

operation group $(P>0.05)$, while there was significant decrease $(P<0.01)$ in operation group after BCCAO, as shown in Table 3.

3.2.2. $r C B F$ after $E A$. After receiving the 4-week treatment, the $\mathrm{rCBF}$ of the EA group significantly increased compared with the model group $(P<0.05)$, though it is still lower than sham group $(P<0.05)$, as shown in Table 4 .

3.3. Effects of EA on $I L-6, T N F-\alpha$, and $I L-1 \beta(\bar{x} \pm s, n g / m L)$. After the 4-week treatment, the IL-6 expression of the model and EA groups increased compared with the sham group, and the difference was statistically significant $(P<0.05)$. Moreover, it increased more remarkably in the EA group, with a significant difference compared with the model group $(P<$ $0.05)$. Also, the expression of TNF- $\alpha$ and IL- $1 \beta$ in the EA and model group increased compared with the sham group; however, they declined in EA group compared with the model group. Particularly, the IL-1 $\beta$ decreased more obviously with a significant difference between EA group and model group $(P<0.05)$ (Table 5 and Figure 3$)$.
3.4. Effects of EA on JAK2 and STAT3. Results showed that 4 weeks after BCCAO, expression of JAK2 and STAT3 mRNA enhanced in model and EA group, particularly in the former; there was significant difference between sham group and model group $(P<0.05)$. However, they were declined in EA group, especially the JAK2 mRNA; there was significant difference between EA group and model group. Western blot showed that expression of JAK2 and STAT3 enhanced in model and EA group, particularly in the model group; there was significant difference between sham group and model group $(P<0.05)$. But they were declined in EA group, especially JAK2; there was significant difference between EA group and model group as shown in Table 6 and Figure 4.

\section{Discussion}

CCI is common in elderly society, which has been identified as a notable risk factor of dementia in patients with cerebrovascular disease $[17,18]$. It is caused by long-term or chronic cerebral hypoperfusion, and it manifests as a group of persistent or progressive cognitive disorders. It is the common pathological basis of vascular dementia, Alzheimer's disease, Binswanger's disease, and so on [1]. Published studies have revealed that individual spatial learning and memory abilities can be achieved only by mutual cooperation of different brain regions, such as hippocampus, striatum, and cerebellum; reduction of $\mathrm{CBF}$ in these regions correlates directly with cognitive impairments [19]. The hippocampus plays a crucial role and its neurons are considered as physiological basis of spatial learning and memory abilities [20]. CCI induces long-term and persistent cerebral hypoperfusion. Thus, the energy metabolism of the hippocampus and other sensitive areas may not be improved continuously, thereby leading to the progressive aggravation of cognitive dysfunction [19]. Some studies reported a diffusely decreased blood flow in the frontal lobe, hippocampus, and other important brain regions 2 years before cognitive function symptoms appear in patients with vascular dementia [21]. Detecting the cerebral blood flow in SD rats of the BCCAO model revealed that the blood flow in the brain cortex and hippocampus significantly decreased $2.5 \mathrm{~h}$ after ischemia. Moreover, it most remarkably decreased in the hippocampus, with a decrease in rate up to $60 \%$ [22]. Meanwhile, with the gradual extension of cerebral hypoperfusion, the neurons in the sensitive areas might gradually appear with ischemia, edema, degeneration and necrosis, depigmentation, and 
TABLE 5: Changes of IL-6, TNF- $\alpha$, and IL- $1 \beta$ in the hippocampus $(\bar{x} \pm s, \mathrm{ng} / \mathrm{ml})$.

\begin{tabular}{lccc}
\hline Groups & IL-6 & TNF- $\alpha$ & IL-1 $\beta$ \\
\hline Sham group & $0.305 \pm 0.019$ & $0.118 \pm 0.022$ & $0.223 \pm 0.021$ \\
Model group & $0.331 \pm 0.015^{\mathrm{a}}$ & $0.170 \pm 0.012^{\mathrm{a}}$ & $0.336 \pm 0.026^{\mathrm{a}}$ \\
EA group & $0.372 \pm 0.011^{\mathrm{ab}}$ & $0.148 \pm 0.020^{\mathrm{a}}$ & $0.279 \pm 0.037^{\mathrm{ab}}$ \\
\hline
\end{tabular}

The protein levels of IL-6, TNF- $\alpha$, and IL-1 $\beta$ were determined by ELISA. Compared with the sham group, ${ }^{\mathrm{a}} P<0.05$; compared with the model group, ${ }^{\mathrm{b}} P<$ 0.05 .

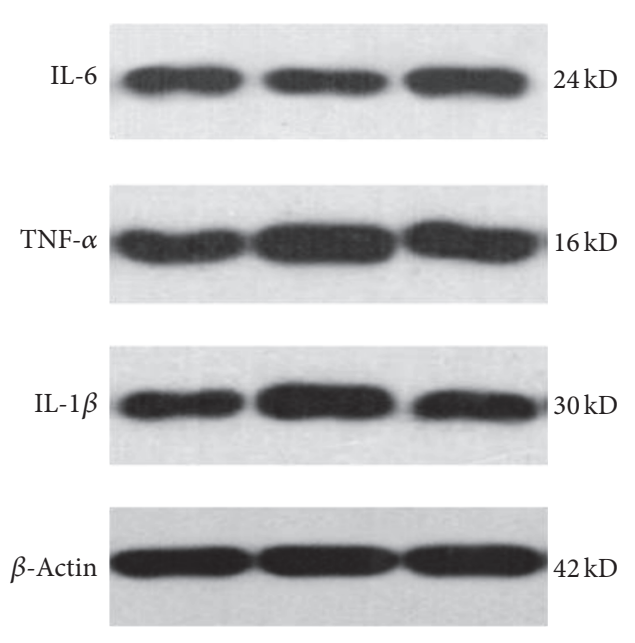

(a)

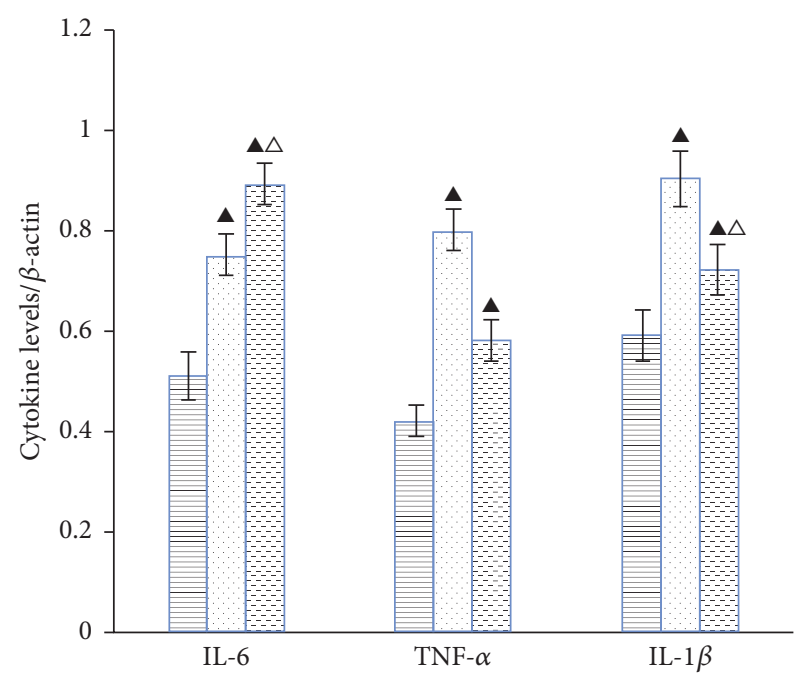

Sham group
Model group
EA group

Figure 3: The protein levels of IL-6, TNF- $\alpha$, and IL-1 $\beta$ in the hippocampus. (a) Representative western blots of proinflammatory cytokines. $\beta$-Actin was used as the loading control. (b) Quantitative analysis of cytokine expression $/ \beta$-actin. ${ }^{\wedge} P<0.05$, versus sham group; ${ }^{\wedge} P<0.05$, versus model group.
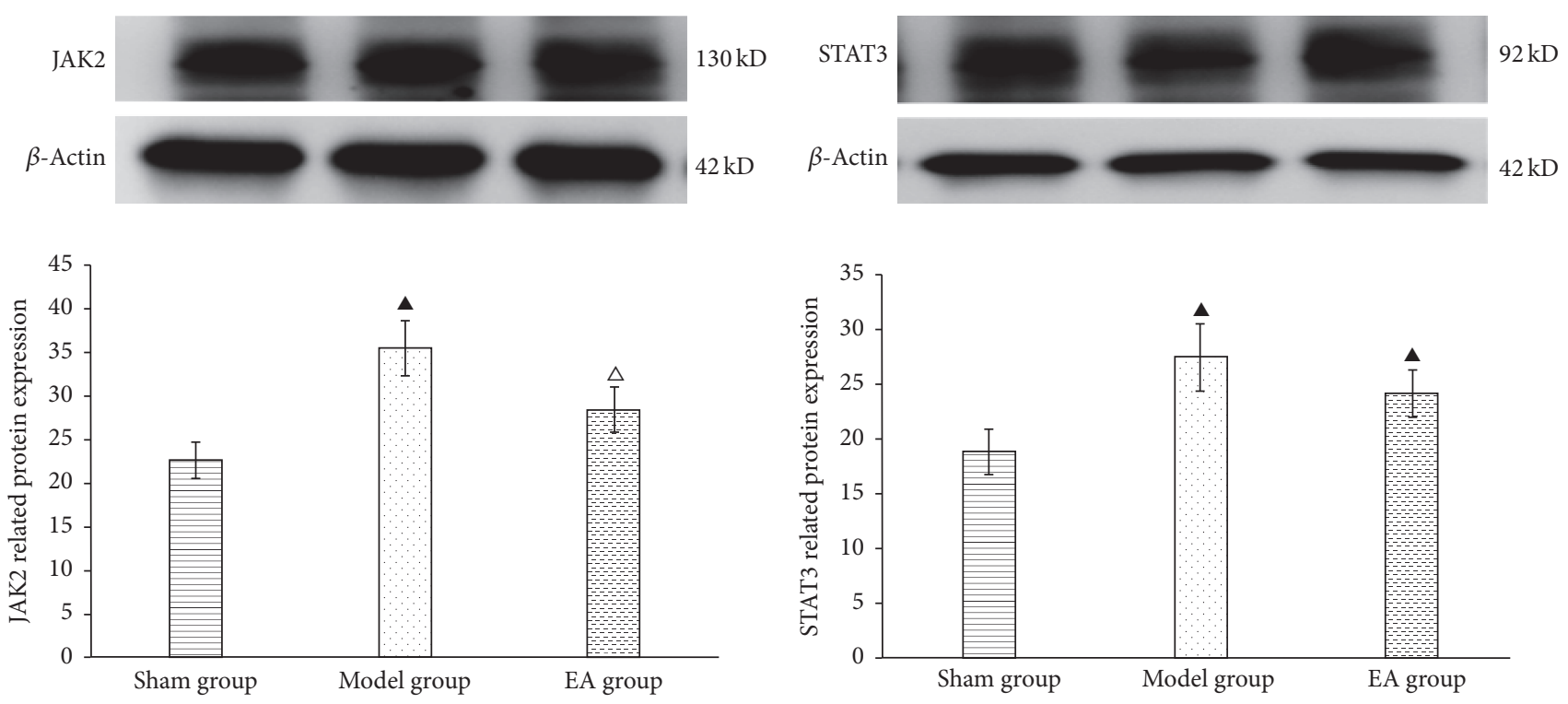

FIGURE 4: Expression of JAK2 and STAT3 protein by western blot in each group. ${ }^{\star} P<0.05$, versus sham group; ${ }^{\triangle} P<0.05$, versus model group. 
TABLE 6: Expression of JAK2/STAT3 mRNA $(\bar{x} \pm s)$.

\begin{tabular}{lcc}
\hline Groups & JAK2 & STAT3 \\
\hline Sham group & $1.125 \pm 0.164$ & $0.795 \pm 0.158$ \\
Model group & $1.528 \pm 0.182^{\mathrm{a}}$ & $1.152 \pm 0.164^{\mathrm{a}}$ \\
EA group & $1.292 \pm 0.162^{\mathrm{b}}$ & $1.002 \pm 0.171^{\mathrm{a}}$ \\
\hline
\end{tabular}

The mRNA levels of JAK2 and STAT3 were detected by RT-PCR. Compared with sham group, ${ }^{\mathrm{a}} P<0.05$; compared with model group, ${ }^{\mathrm{b}} P<0.05$.

other pathological changes [23]. As reported, this study also showed that the $\mathrm{rCBF}$ of the operation group significantly decreases after BCCAO, and the control level dropped sharply and reduced to $35-45 \%$ in the cerebral cortex and $60 \%$ in the hippocampus in the acute phase. Then, the CBF values started to increase gradually from the first week, which is a phase of chronic hypoperfusion closely resembling human, but it remained significantly lower than sham group [7]. After receiving the 4-week EA treatment, as showed in other studies [24], the rCBF of EA group improved statistically compared with the model group, though it was still lower than that of the sham group.

Inflammatory response is well documented and plays a key role in CCI injury $[11,25]$, which includes inflammatory immune cells, inflammatory immune factors, and adhesion molecules. Among inflammatory immune factors, IL-1 $\beta$, TNF- $\alpha$, and IL- 6 are closely associated with the central nervous system and ischemic injuries, which can lead to demyelination, oligodendrocyte apoptosis, thrombosis, vascular proliferation, leukocyte infiltration, and blood-brain barrier disruption [26]. Of these, IL-1 is an important inflammatory factor triggering immune and inflammatory response, which also can aggravate ischemic brain injury. Particularly, IL-1 $\beta$ is closely associated with the pathogenesis of cerebral ischemia [27]. IL-1 $\beta$ also has a synergistic effect with TNF- $\alpha$ to further exacerbate brain damage $[28,29]$ and cerebral ischemia can upregulate TNF- $\alpha$ and participate in the pathological process of brain injury [30], while TNF- $\alpha$ can promote the release of IL- $1 \beta$ and other cytokines. IL- 6 plays a complex role in the central nervous system; its role in cerebral ischemic injury is still controversial [31, 32]. Some believed that IL-6 is beneficial in cerebral ischemia [33], but some deemed it may exacerbate brain injury [32]. However, the majority of available studies indicate that IL- 6 exerts a neuroprotective effect and promotes the recovery of neurological functions. Inflammatory response can be propagated and amplified by the JAKSTAT signaling pathway [34]. JAK-STAT signaling has been reported to be involved in inflammatory responses [35]. As JAK2 is upstream STAT3, present study shows that although normal levels of JAK2/STAT3 activation are essential for cellular functions, excess STAT3 activation is detrimental to brain, and prevention of ischemic-induced JAK2/STAT3 phosphorylation is neuroprotective [36]. The findings of our study also revealed that EA could modulate the expression of IL-1 $\beta /$ TNF- $\alpha /$ IL- 6 and downregulate the excess activation of JAK2/STAT3 in CCI rats.
EA has been proved to be capable of improving cognitive function, which might be associated with regulating cell proliferation in different brain regions [15] and activating adenosine monophosphate-activated kinase (AMPK) [37]. In addition, EA showed anti-inflammatory effects including attenuating the activation of microglia via the TLR4/NF- $\kappa \mathrm{B}$ signaling pathway [38] and the TLR2, JNK/c-Jun pathways, modulating the release of TNF- $\alpha$, IL-1, and IL-6 in MACO rats [39]. Our research was consistent with it. Results showed that EA on DU20 and DU14 is prone to improve cognitive function modulating the release of inflammatory immune factors including TNF- $\alpha$, IL-1 $\beta$, and IL- 6 which may be achieved through improving the rCBF and depressing the excess activation of JAK2/STAT3 signaling pathway.

Of course, until now, only initial conclusions have been drawn and we will do further research upon the following goals: firstly, classical CCI model was evaluated by the water maze and pathological section in 3-6 weeks after BCCAO operation and this study emphasizes intervention in early stages. Therefore, in addition to $\mathrm{rCBF}$, it is imperative to find a new evaluation method. Secondly, it is meaningful to investigate the effect of EA on phosphorylation of JAK2/STAT3 pathway. Thirdly, increasing a blocking group and blocking the JAK/STAT pathway with inhibitor AG490 are necessary to clarify the effect of EA.

\section{Conclusions}

In summary, our present evidence demonstrated that EA on DU20 and DU14 can improve cognitive function after CCI. This may be achieved by increasing $\mathrm{rCBF}$, downregulating the excess activation of JAK2 and STAT3, and mediating IL6 , TNF- $\alpha$, and IL- $1 \beta$ levels thus attenuating inflammatory injury. Though the protective mechanisms have not been fully elucidated, the data demonstrate that EA is a promising approach to treat chronic cerebral ischemia.

\section{Conflicts of Interest}

All authors declare that there are no conflicts of interest regarding the publication of this paper.

\section{Acknowledgments}

The authors sincerely thank all the volunteers for their participation in this study. This work was supported by the Key Science and Technology Innovation Team of Zhejiang Province (no. 2013TD15); the National Natural Science Foundation of China (no. 81503645); the Natural Science Foundation of Zhejiang Province of China (nos. LY16H270004 and Y205389).

\section{References}

[1] M.-F. Dubois and R. Hébert, "The incidence of vascular dementia in Canada: a comparison with Europe and East Asia," Neuroepidemiology, vol. 20, no. 3, pp. 179-187, 2001. 
[2] C. Iadecola, "The pathobiology of vascular dementia," Neuron, vol. 80, no. 4, pp. 844-866, 2013.

[3] Y. Zhao, J.-H. Gu, C.-L. Dai et al., "Chronic cerebral hypoperfusion causes decrease of O-GlcNAcylation, hyperphosphorylation of tau and behavioral deficits in mice," Frontiers in Aging Neuroscience, vol. 6, article 10, 2014.

[4] J. Bang, W. K. Jeon, I. S. Lee, J.-S. Han, and B.-Y. Kim, "Biphasic functional regulation in hippocampus of rat with chronic cerebral hypoperfusion induced by permanent occlusion of bilateral common carotid artery," PLOS ONE, vol. 8, no. 7, Article ID e70093, 2013.

[5] Z.-Y. Cai, Y. Yan, and R. Chen, "Minocycline reduces astrocytic reactivation and neuroinflammation in the hippocampus of a vascular cognitive impairment rat model," Neuroscience Bulletin, vol. 26, no. 1, pp. 28-36, 2010.

[6] J. T. Neumann, C. H. Cohan, K. R. Dave, C. B. Wright, and M. A. Perez-Pinzon, "Global cerebral ischemia: synaptic and cognitive dysfunction," Current Drug Targets, vol. 14, no. 1, pp. 20-35, 2013.

[7] E. Farkas, P. G. M. Luiten, and F. Bari, "Permanent, bilateral common carotid artery occlusion in the rat: a model for chronic cerebral hypoperfusion-related neurodegenerative diseases," Brain Research Reviews, vol. 54, no. 1, pp. 162-180, 2007.

[8] S.-Q. Du, X.-R. Wang, L.-Y. Xiao et al., "Molecular mechanisms of vascular dementia: what can be learned from animal models of chronic cerebral hypoperfusion?" Molecular Neurobiology, 2016.

[9] S.-K. Kim, K.-O. Cho, and S. Y. Kim, "White matter damage and hippocampal neurodegeneration induced by permanent bilateral occlusion of common carotid artery in the rat: comparison between Wistar and Sprague-Dawley strain," The Korean Journal of Physiology and Pharmacology, vol. 12, no. 3, pp. 8994, 2008.

[10] C. S. Nicolas, M. Amici, Z. A. Bortolotto et al., "The role of JAKSTAT signaling within the CNS," JAKSTAT, vol. 2, no. 1, Article ID e22925, 2013.

[11] K. M. Lee, J. H. Bang, J.-S. Han et al., "Cardiotonic pill attenuates white matter and hippocampal damage via inhibiting microglial activation and downregulating ERK and p38 MAPK signaling in chronic cerebral hypoperfused rat," BMC Complementary and Alternative Medicine, vol. 13, article 334, 2013.

[12] T. Urabe, "Molecular mechanism and new protective strategy for ischemic white matter damages," Clinical Neurology, vol. 52, no. 11, pp. 908-910, 2012.

[13] H. S. Han and M. A. Yenari, "Cellular targets of brain inflammation in stroke," Current Opinion in Investigational Drugs, vol. 4, no. 5, pp. 522-529, 2003.

[14] C. X. Wang and Y. Z. Sun, "Effects of scalp point therapy on expression of endothelial growth factors in hippocampus of rats with chronic cerebral ischemia," Acupuncture Research, vol. 37, no. 5, pp. 375-379, 2012.

[15] H. Y. Cheng, J. C. Yu, Z. G. Jiang et al., "Acupuncture improves cognitive deficits and regulates the brain cell proliferation of SAMP8 mice," Neuroscience Letters, vol. 432, no. 2, pp. 111-116, 2008.

[16] J.-W. Ni, H. Ohta, K. Matsumoto, and H. Watanabe, "Progressive cognitive impairment following chronic cerebral hypoperfusion induced by permanent occlusion of bilateral carotid arteries in rats," Brain Research, vol. 653, no. 1-2, pp. 231-236, 1994.

[17] M. Cankurtaran, B. B. Yavuz, E. S. Cankurtaran, M. Halil, Z. Ulger, and S. Ariogul, "Risk factors and type of dementia: vascular or Alzheimer?" Archives of Gerontology and Geriatrics, vol. 47, no. 1, pp. 25-34, 2008.

[18] P. B. Gorelick, "Risk factors for vascular dementia and Alzheimer disease," Stroke, vol. 35, no. 11, supplement 1, pp. 26202622, 2004.

[19] J. S. Meyer, R. L. Rogers, B. W. Judd, K. F. Mortel, and P. Sims, "Cognition and cerebral blood flow fluctuate together in multiinfarct dementia," Stroke, vol. 19, no. 2, pp. 163-169, 1988.

[20] S. Rosi, M. Andres-Mach, K. M. Fishman, W. Levy, R. A. Ferguson, and J. R. Fike, "Cranial irradiation alters the behaviorally induced immediate-early gene Arc (activity-regulated cytoskeleton-associated protein)," Cancer Research, vol. 68, no. 23, pp. 9763-9770, 2008.

[21] R. L. Rogers, J. S. Meyer, K. F. Mortel, R. K. Mahurin, and B. W. Judd, "Decreased cerebral blood flow precedes multi-infarct dementia, but follows senile dementia of Alzheimer type," Neurology, vol. 36, no. 1, pp. 1-6, 1986.

[22] Y. Shu, H. Zhang, and J. J. Zhang, "Advances in experimental research of chronic cerebral hypoperfusion," Chinese Journal of Cerebrovascular Diseases, vol. 4, no. 3, pp. 199-205, 2010.

[23] L. Sun, J. Wu, S. C. Wang, and Y. Zhang, "Cerebral blood flow and apoptosis in rats with vascular dementia," Chinese Journal of Geriatric Cardiovascular and Cerebrovascular Diseases, vol. 3, no. 6, pp. 409-411, 2001.

[24] X. Zhang, B. Wu, K. Nie, Y. Jia, and J. Yu, "Effects of acupuncture on declined cerebral blood flow, impaired mitochondrial respiratory function and oxidative stress in multi-infarct dementia rats," Neurochemistry International, vol. 65, no. 1, pp. 23-29, 2014.

[25] N. G. Frangogiannis, "Chemokines in ischemia and reperfusion," Thrombosis and Haemostasis, vol. 97, no. 5, pp. 738-747, 2007.

[26] W. Duan, L. Gui, Z. Zhou et al., "Adenosine A2A receptor deficiency exacerbates white matter lesions and cognitive deficits induced by chronic cerebral hypoperfusion in mice," Journal of the Neurological Sciences, vol. 285, no. 1-2, pp. 39-45, 2009.

[27] H. Boutin, R. A. LeFeuvre, R. Horai, M. Asano, Y. Iwakura, and N. J. Rothwell, "Role of IL- $1 \alpha$ and IL- $1 \beta$ in ischemic brain damage," Journal of Neuroscience, vol. 21, no. 15, pp. 5528-5534, 2001.

[28] P. D. Hurn, S. Subramanian, S. M. Parker et al., “T- and B-celldeficient mice with experimental stroke have reduced lesion size and inflammation," Journal of Cerebral Blood Flow and Metabolism, vol. 27, no. 11, pp. 1798-1805, 2007.

[29] W. Pan and A. J. Kastin, "Tumor necrosis factor and stroke: role of the blood-brain barrier," Progress in Neurobiology, vol. 83, no. 6, pp. 363-374, 2007.

[30] E. Pinteaux, N. J. Rothwell, and H. Boutin, "Neuroprotective actions of endogenous interleukin-1 receptor antagonist (IL1ra) are mediated by glia," GLIA, vol. 53, no. 5, pp. 551-556, 2006.

[31] O. Herrmann, V. Tarabin, S. Suzuki et al., "Regulation of body temperature and neuroprotection by endogenous interleukin6 in cerebral ischemia," Journal of Cerebral Blood Flow and Metabolism, vol. 23, no. 4, pp. 406-415, 2003.

[32] C. J. Smith, H. C. Emsley, C. M. Gavin et al., "Peak plasma interleukin- 6 and other peripheral markers of inflammation in the first week of ischaemic stroke correlate with brain infarct volume, stroke severity and longterm outcome," BMC Neurology, vol. 4, article 2, 2004.

[33] S. M. Allan and N. J. Rothwell, "Cytokines and acute neurodegeneration," Nature Reviews Neuroscience, vol. 2, no. 10, pp. 734744, 2001. 
[34] J. J. O’Shea, M. Gadina, and R. D. Schreiber, "Cytokine signaling in 2002: new surprises in the Jak/Stat pathway," Cell, vol. 109, no. 2, supplement 1, pp. S121-S131, 2002.

[35] O. S. Kim, E. J. Park, E.-H. Joe, and I. Jou, "JAK-STAT signaling mediates gangliosides-induced inflammatory responses in brain microglial cells," The Journal of Biological Chemistry, vol. 277, no. 43, pp. 40594-40601, 2002.

[36] I. Satriotomo, K. K. Bowen, and R. Vemuganti, "JAK2 and STAT3 activation contributes to neuronal damage following transient focal cerebral ischemia," Journal of Neurochemistry, vol. 98, no. 5, pp. 1353-1368, 2006.

[37] W. Dong, W. Guo, X. Zheng et al., "Electroacupuncture improves cognitive deficits associated with AMPK activation in SAMP8 mice," Metabolic Brain Disease, vol. 30, no. 3, pp. 777784, 2015.

[38] B. Han, Y. Lu, H. Zhao, Y. Wang, L. Li, and T. Wang, "Electroacupuncture modulated the inflammatory reaction in MCAO rats via inhibiting the TLR4/NF- $\kappa$ B signaling pathway in microglia," International Journal of Clinical and Experimental Pathology, vol. 8, no. 9, pp. 11199-11205, 2015.

[39] H.-Y. Lin, C.-H. Tang, J.-H. Chen et al., "Peptidoglycan induces interleukin-6 expression through the TLR2 receptor, JNK, cJun, and AP-1 pathways in microglia," Journal of Cellular Physiology, vol. 226, no. 6, pp. 1573-1582, 2011. 


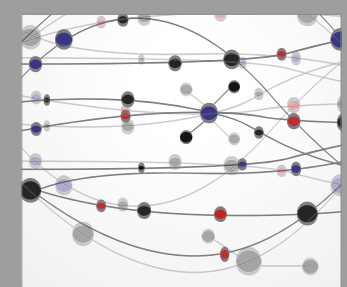

The Scientific World Journal
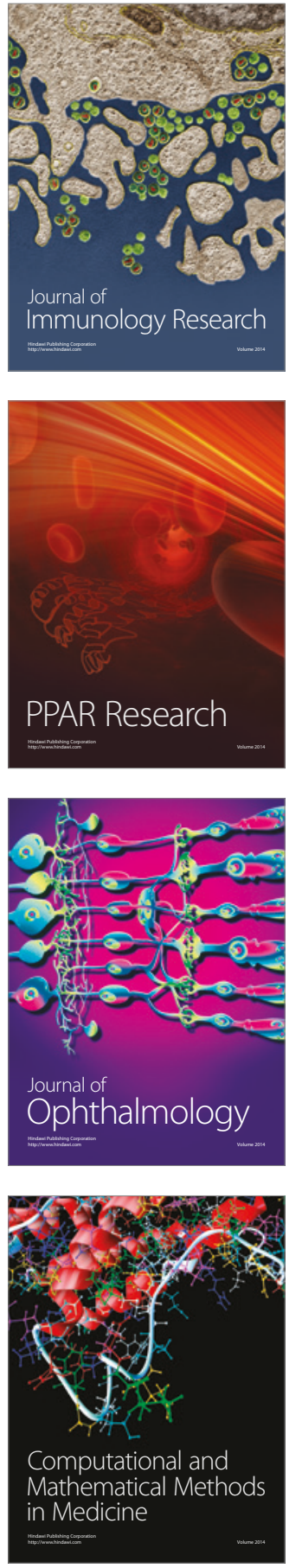

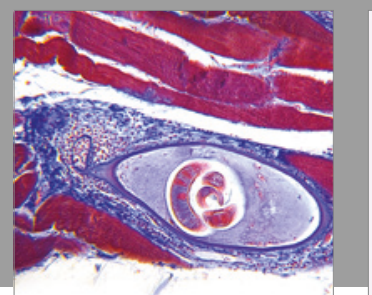

Gastroenterology Research and Practice
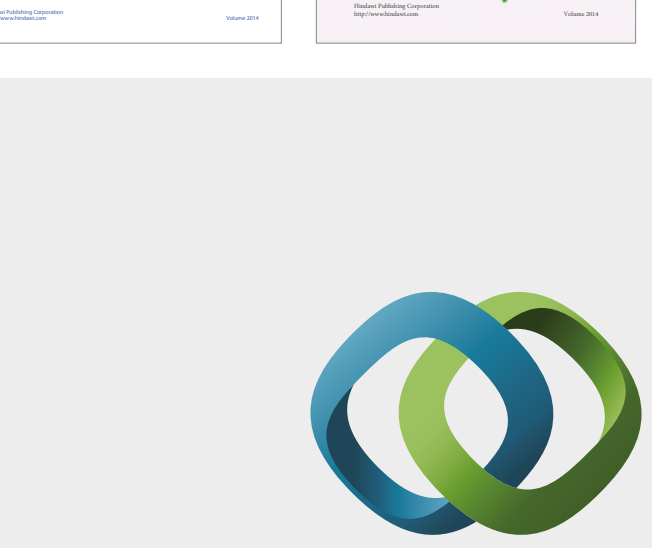

\section{Hindawi}

Submit your manuscripts at

https://www.hindawi.com
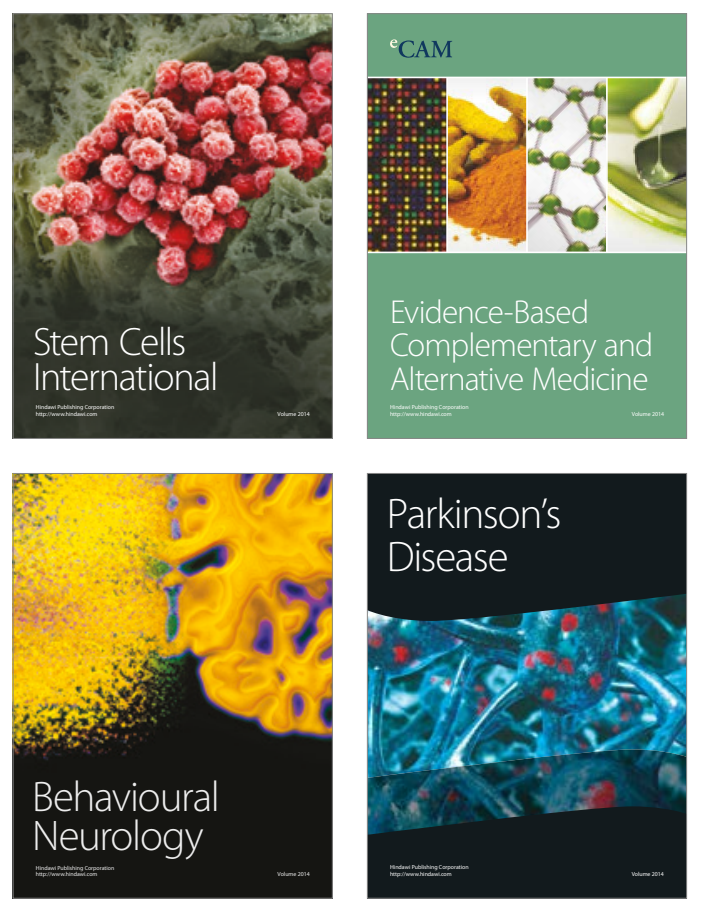
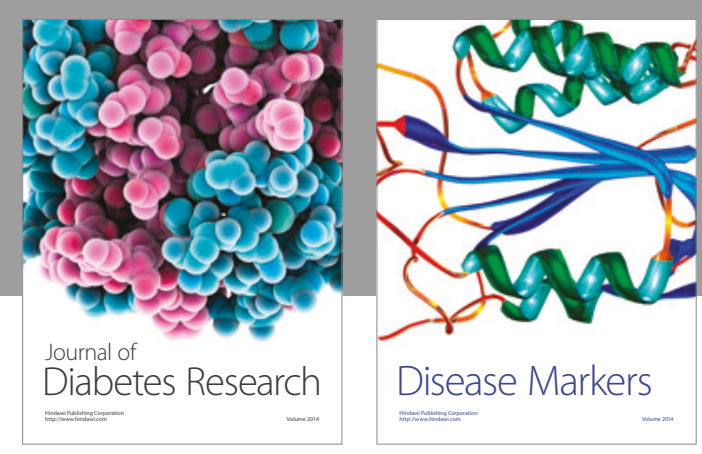

Disease Markers
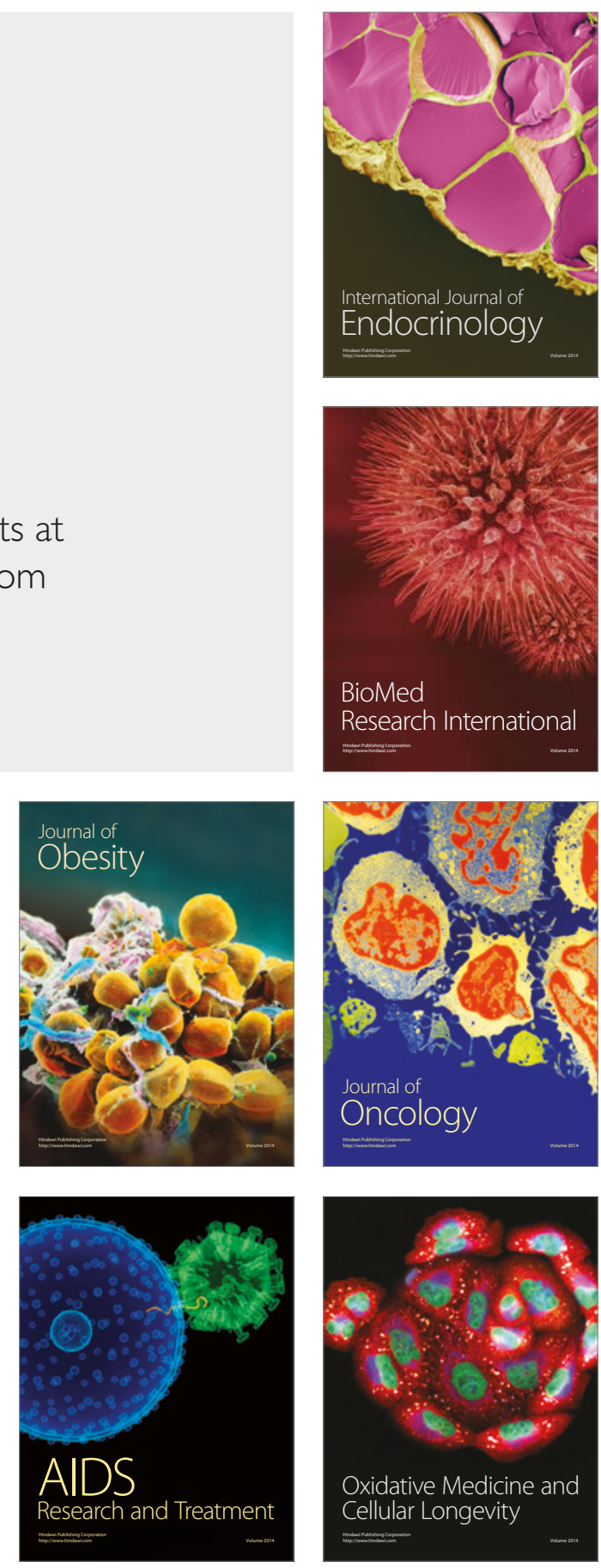